\title{
Towards designing Brain-Computer Interfaces in terms of User-Profiles, Neurophysiological Factors and User Experience
}

\section{Hacia el diseño de Interfaces Cerebro-Computadora en términos del Perfil del Usuario, Factores Neurofisiológico y Experiencia del Usuario}

\author{
L. M. Alonso-Valerdi, V. R. Mercado-García \\ Tecnológico de Monterrey, Escuela de Ingeniería y Ciencias
}

\begin{abstract}
Brain-computer interfaces (BCIs) are technology in development that attempt to establish interaction between individuals and their surroundings by modulating their neural activity. One of the most common strategies to modulate neural activity is motor imagery (MI). However, research on MI-based BCIs has been mostly carried out on the system-related part, whereas the user-related part has been relatively ignored. Thus far, up to $30 \%$ of users cannot gain control of BCI, while the remaining ones reach modest performance. The exclusion of users in the system design has possibly led to this outcome. Therefore, the aim of this paper is to establish a mixed method based on interactive design principles and in line with (1) user-profile, (2) psychological and (3) neurophysiological factors, (4) BCI technical issues and (5) user-experience. Although some of these elements have been previously discussed, their integration and application are seldom considered during investigation.
\end{abstract}

KEYwords: Brain-Computer Interfaces; Neurophysiological Factors; User-Experience; User-Profile 


\section{RESUMEN}

Las interfaces cerebro-computadora (ICC) son tecnología en desarrollo que intenta establecer interacción entre un individuo y su entorno a través de la modulación de su actividad neuronal. Una de las estrategias más usadas para modular la actividad neuronal ha sido la imaginación motora. Sin embargo, la investigación en ICC controladas por imaginación motora ha sido desarrollada mayoritariamente en términos del sistema, donde el usuario es generalmente ignorado. A la fecha, hasta el 30\% de los usuarios no pueden controlar un sistema ICC basado en imaginación motora, mientras que el resto de los usuarios alcanzan un desempeño moderado. La exclusión de los usuarios en el diseño del sistema, posiblemente ha llevado al bajo índice de adaptación entre el sistema y el usuario. En base a esta evidencia, el objetivo de este artículo es establecer un método mixto sustentado en principios de diseño interactivo y considerando cinco elementos: (1) perfil del usuario, (2) factores psicológicos y (3) neurofisiológicos, (4) factores técnicos y (5) experiencia del usuario. Aunque todos estos elementos han sido discutidos previamente, su integración y aplicación son muy poco frecuentes durante la investigación.

PALABRAS CLAVE: Interfaces Cerebro-Computador; Factores Neurofisiológicos; Experiencia del Usuario; Perfil del Usuario

\section{Correspondencia}

DESTINATARIO: Luz María Alonso Valerdi

INSTITUCIÓN: Tecnológico de Monterrey,

Escuela de Ingeniería y Ciencias

DIRECCIÓN: Ave. Eugenio Garza Sada \#2501,

Col. Tecnológico, C. P. 64849, Monterrey,

Nuevo León, México

CORREO ELECTRÓNICO: Im.aloval@tec.mx

\section{Fecha de recepción:}

12 de junio de 2018

Fecha de aceptación:

24 de febrero de 2019 


\section{INTRODUCTION}

A brain-computer interface (BCI) is a system that attempts to establish interaction between individuals and their surroundings by translating brain signals into control commands. Brain signals are modulated by control tasks, which can be of two types: endogenous and exogenous. Endogenous control tasks refer to mental rehearsal such as imagination of specific movements without actual execution (i.e., motor imagery) or mental rotation of 3D objects. On the other hand, exogenous ones regard the focus of attention towards visual, auditory or haptic stimuli ${ }^{[1]}$.

One of the most commonly used control task has been motor imagery (MI) due to the natural way to perform the task and the sense of agency ${ }^{[2]}$. Research on MI based BCIs has been mostly carried out on the system-related part. However, the user-related part has been relatively ignored. Thus far, a considerable portion of naïve users have been classified as low aptitude users because they do not achieve adequate system performance (i.e., detection of at least $70 \%$ of neural patterns associated with MI). According to ${ }^{[3,4]}$, researchers in the field have reported portions of low aptitude naïve users from $40 \%$ to $60 \%$ of their sample in use. Similarly, it was argued in ${ }^{[2]}$ that between $15 \%$ and $30 \%$ of naïve users could not control BCI at all, whereas the remaining users only reached modest performance. It is also important to note that performance variation is not only present across different users, but also the same user can present high performance variations ${ }^{[5,6]}$. The exclusion of users in the system design has possibly led to this outcome.

The system design in terms of the user, rather than in terms of the system per se, is well-known as interaction design ${ }^{[7]}$. In the light of aforementioned evidence, it seems plausible that BCI community has been failing in developing interactive designs of MI based BCIs. Therefore, the present paper aims to offer some important insights into interactive design of MI based BCIs by raising three fundamental questions: (1) who is going to use the system? (2) how is the system going to be operated? and (3) where is the system going to be used?

The first question concerns the user. So far, users have been seen as entities with no history. However, every individual has different anatomy, body functioning, lifestyles, habits, skills, and interests. It has been shown that all of these factors (and many others) determine the nature of the brain activity, and the feasibility of being modulated through $\mathrm{MI}{ }^{[2]}$. As a result, the first step towards prototyping interactive designs must be to build up user-profiles.

The second question relates to the control tasks. In MI based BCIs, the term "control task" refers to generate motor mental images that could produce distinguishable brain patters over the scalp. MI related control tasks are a skill that must be acquired by training, and which also depends on the user-profile. Furthermore, imaginary movements are predicted and produced in line with motor repertoires inherited at birth and built along lifetime ${ }^{[8]}$. Thus, psychological and neurophysiological factors of MI-related control tasks must be considered as well.

The third question is associated with the context, which can be physical, social, and organizational [9]. Physical context may limit the number of tasks undertaken at a time due to environmental distractors, and it could also push users to explore different ways to solve the same problem at different times. Social context can determine user guidance, and even the acceptability of certain designs. Organizational context is associated with the availability of easy-to-access and easy-to-digest information. All these factors strongly influence the user performance, but such influence might be quantifiable, once a prototype had been tested by end-users in real working conditions ${ }^{[10]}$. Consequently, user-experience (UX) must be obtained to redesign MI based BCIs in line with end-user requirements. 


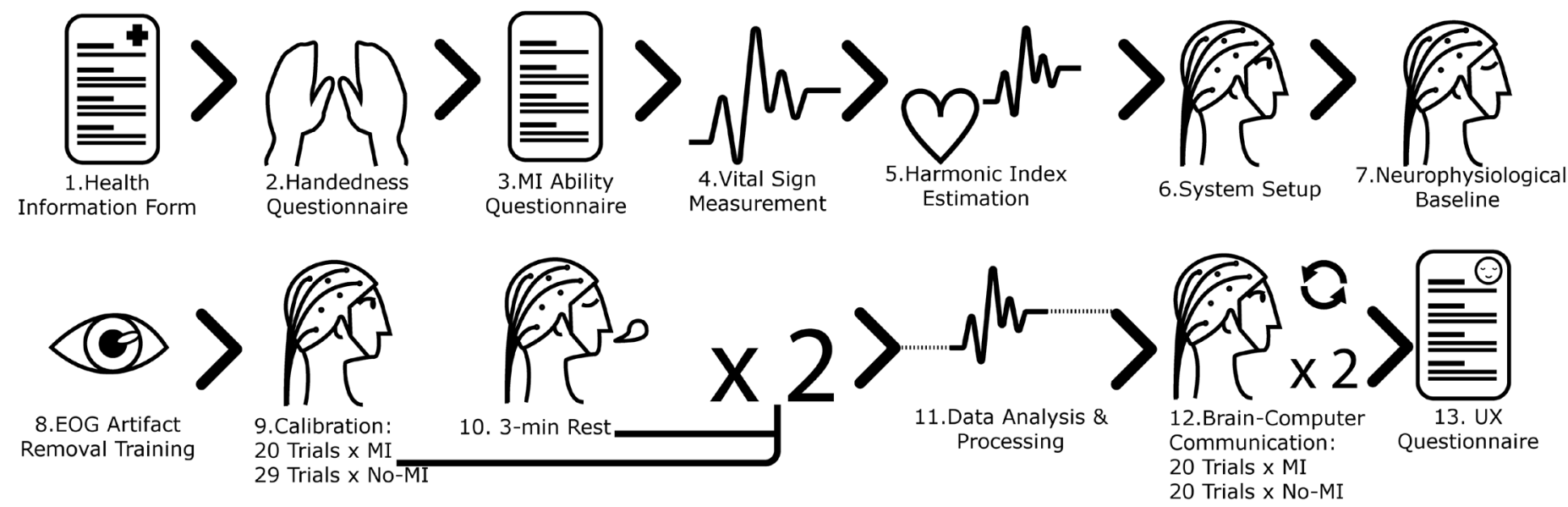

FIGURE 1. Methodological workflow to design MI based BCls in line with user-profile, psychological factors, neurophysiological evaluation, and UX undergone throughout the brain-computer communication.

In the light of the above discussion, the aim of this paper is to establish the initial steps towards new methods for designing easy-to-learn, effective-to-use and enjoyable MI based BCIs on the basis of (1) user-profiles, (2) psychological and neurophysiological factors associated with MI control tasks, and (3) UX so as to improve individual user performances, and increase the number of qualified users.

\section{MIXED METHOD FOR BCIS}

BCI community has been always surrounded with hopes and expectations of providing a reliable way of communication between the human brain and a computer system. This research field has been getting more robust in important areas ranging from pattern recognition to electrode (transducer to record non-invasively electrical body activity) and material improvements, along with human-computer interaction techniques. However, methodologies are often skipped or dimly presented in the state-of-the-art publications [11], leading to a great difficulty or impossibility of replicating experiments in this area. The purpose of this paper is to propose a mixed methodological procedure in line with user-profiles, neuro-evaluations (both psychological and physiological), and UX. The step-bystep proposed method is depicted in Figure 1. As can be seen from the figure, the method involves 12 steps, which are encompassed under five stages: (1) user-profile, (2) psychological and (3) neurophysiological evaluation, (4) technical issues in BCI and (5) UX.

\section{User-profile}

As can be seen, life history, life style, and living conditions of users affect MI based BCI performance, and from here, the relevance of create user-profiles. A userprofile can be helpful to define the system structure to exploit user strengths, and overcome user weaknesses. It is proposed to build up user-profiles according to health information, lifestyle, and handedness.

\section{Health information and lifestyle}

Health information and lifestyle can reveal the facility or difficulty of users to produce specific imaginary movements at the time of controlling a MI based BCI $[12,13]$. By way of illustration, an online questionnaire concerning physical and medical condition, sleeping and nutrition habits, alcohol consumption, smoking habits, and hormonal conditions has been attached to this $\underline{\text { paper }}^{i}$. In addition, vital signs, including body temperature, breathing frequency, blood pressure and heart rate should be registered. Particularly, heart rate is very relevant to adjust the frequency band of sensorimotor rhythms (brain patterns related to MI) according to the neurobiology aspects of the user. It is com- 
mon practice to make use of the pre-defined frequency bands. However, it is well-established that neural oscillations change over lifecycle. By using heart rate and applying the brain-body coupling theory can be estimated neural resonance rhythms of each user ${ }^{[14]}$.

\section{Handedness}

Imaginary movements stimulate the same neurocognitive and learning processes as actual ones. Thus, it is expected the relevance of the dominant hand of BCI users. In ${ }^{[15]}$, this point is clearly illustrated. Researchers found that MI practice with the dominant hand resulted in larger and more robust improvements in movement speed comparted with MI practice with the non-dominant one. As it is very relevant to determine participant handedness, standard handedness evaluation ${ }^{\text {ii }}$ related to hand preference to carry out daily tasks such as writing, drawing, throwing, striking a match, opening a box, eating, toothbrushes, brooms, and scissoring are necessary. This test is based on ${ }^{[16]}$. Once the test has been completed, a laterality index is obtained which determines the percentile of handedness for the participant. By identifying the dominant hand, a well-chosen selection of control tasks for brain-computer interfacing may be offered at the prototype stage.

\section{Psychological factors}

To date, the psychological factors associated with MI tasks have been (1) perception of an imagination task, (2) visual-motor coordination, (3) level of attention, (4) spatial abilities, (5) intelligence, (4) personality traits, (5) psychological well-being, (6) motivation, and (7) mood ${ }^{[17]}$. All these factors may be encompassed under two categories: MI process and motivation. On the one hand, the imagination strategy to elicit motor mental images and the level of difficulty of the motor task (e.g., duration, velocity, complexity) are factors that affect whether users achieve to control their brain activity through MI control tasks ${ }^{[18,3]}$. On the other hand, the level of user engagement at the moment of the brain-computer communication has always been critical. Motivation is a very complex factor that cannot be only influenced by entertainment and excitement as has been done via virtual environments, but it can also be modified via fatigue and frustration ${ }^{[19]}$.

On this evidence, objective (e.g., psychophysiological monitoring) and subjective (e.g., questionnaires) evaluations of the user mental state along the experimental procedures should be obligatory. For instance, several MI questionnaires have been provided to measure movement imaginary abilities. There is no certainty about MI existence since it exclusively depends on the user intentions, and he/she is the only one who knows what and how is happening internally. Therefore, a way to give an insight into user mental projections is to evaluate movement imagery ability by means of official questionnaires ${ }^{\text {iii }}$ [20, 21, 22]. Note that these questionnaires have been also used as a simple method to detect BCI illiteracy in MI based BCIs ${ }^{[23]}$. Unfortunately, it is still occasional to see that researchers in the field do not report to have made use of them. Furthermore, not only the use, but also the analysis of the questionnaire outcomes should be obligatory.

\section{Neurophysiological factors}

As it has been herein discussed, MI is an individual capacity to image movements and a skill that needs to be acquired. Brain-computer communication depends on the fertility of imaginary movements selected as control tasks, since the voluntary control of brain activity is achieved by the effectiveness of MI mechanisms employed by the user. Similar to other authors, it is herein considered that brain-computer communication will breakdown, even applying the most sophisticated computational algorithms, if user is unable to show great motor imagination.

Hitherto, it has been commented about personal attributes and psychological factors that determine the nature of MI. However, the genetic influence and the evolution of brain activity along the life-cycle 
could be even more determinant. A notable example is the theory proposed by Klimesch, who suggested that brain-body interactions can be described as a complex system that couples and decouples on the basis of a specific harmonic frequency: heart rate ${ }^{[14]}$. This theory establishes that heart rate, which is known to vary with body size, age, and sex, is the basic frequency and the scaling factor for all other frequency domains: (1) brainstem oscillations that trigger inhaling and exhaling, (2) breathing frequency, and (3) brain oscillations. The work of Klimesch confirms that brain oscillations are determined by basic biological factors; and moreover, that they can be adjusted according to the individual heart rate. In a similar case, brain oscillations in alpha and beta bands over the sensorimotor cortex at resting state have been used to anticipate the user performance in MI based BCIs ${ }^{[24]}$. In the same line of thinking, brain oscillations in high theta, low alpha, and gamma frequency bands have been also proposed as neurophysiological predictors of MI suitability as control task ${ }^{[3,25]}$. Besides, brain activity has not only been used to characterize individuals as potential BCI users, but the electrophysiological activity of nervous system has also been subject of study. As a case in point, MI ability through electro-dermal activity was assessed in ${ }^{[26]}$. As it can be seen, abundant and strong neurophysiological evidence has been accumulated to show that feasibility and reliability of interactive systems such as BCIs depend on biological and physiological aspects of users. Additionally, all this information must be utilized (1) to estimate neural features used to detect MI related control tasks, (2) to evaluate new training protocols, (3) to quantify the learning level of user, and (4) to assess new multi-sensorial feedback strategies such as tactile stimulation.

\section{Vital signs and harmonic index}

In the light of the above discussion, vital signs, including body temperature, breathing frequency, blood pressure and heart rate should be registered, before an BCI experimental procedure. Particularly, heart rate is very relevant to adjust the frequency band of sensorimotor rhythms according to the neurobiology aspects of the user. It is common practice to make use of the pre-defined frequency bands. However, it is well-established now that brain oscillations change over life-cycle, and the brain-body coupling theory can help to localize "real" neural resonance of each user ${ }^{[14]}$.

\section{Neurophysiological predictor}

In addition to vital signs, recording of brain activity at rest before a BCI experimental procedure should be also considered. Some parameters that can be determined by using this recording are individual alpha frequency and the neurophysiological predictor index to categorize users with low or high MI aptitudes. As it was aforementioned, such user categorization can be helpful to test the effectiveness of new training protocols or new feedback strategies. To obtain the individual alpha frequency, it can be employed the method proposed in [27], where two three-minute-long recordings in eyes-closed and eyes-open conditions are necessary. Individual alpha frequency is specifically obtained from two occipital recording sites over the scalp. To estimate the neurophysiological predictor index, the method proposed in ${ }^{[28]}$ has demonstrated to be one of the most effective. The method requires a two-minute-long recording in eyes-closed condition. The general procedure consists in calculating the power spectral density of two central recording sites over the scalp ${ }^{[24]}$. Implementations can be found in public sites such as GitHub ${ }^{[29]}$.

\section{BCI: Technical issues}

The discussion of BCI design is beyond the scope of this paper. However, there are some critical technical issues that are often ignored, and determinant to establish brain-computer communication.

\section{Electrode montage}

The commonly used method to record brain activity is Electroencephalography (EEG). An EEG signal is typically measured between two electrodes, and one of 
them is used as referencing point. However, it is frequently overlooked the sensitiveness of the referencing electrode to the electrical brain activity. It is common practice to place the referencing electrode on a hypothetically "inactive" area of the scalp, or on another part of the body such as the left or right earlobe, or thereof. When a particular lobe is chosen as a reference, the EEG amplitude decreases on the electrodes that are close to the referencing electrode. When linked earlobes is chosen, the asymmetry effect of using one lobe referencing electrode is avoided. However, the link wire between two earlobe referencing electrodes affects intracranial currents that produce the EEG potentials. This inconvenient effect also produces a distortion on the EEG recording [30, 31, 32, 33]. The referencing issue on EEG has been analyzed by the BCI community. A case in point, the estimation of the optimal location of the referencing electrode for MI based BCI using functional magnetic resonance imaging was proposed in ${ }^{[34]}$. In such method, a proper referencing point was estimated to maximize the differentiation between two mental tasks: imagination of left and right finger movements. Authors found that the best reference to discriminate two MI related tasks was FCz, a recording site localized on the supplementary motor area.

The electrode montage determines the spatial filtering of the EEG signals, which in turn maximizes the neuro-mechanisms of interest. This is why the selection of an optimal electrode montage, along with exact electrode location, is significant. On the one hand, electrode positioning must be in line with an imaginary transversal line drawn from nasion to inion, and an imaginary longitudinal line drawn from left to right pre-auricular points. The crossing point of these two imaginary lines should be the recording site $\mathrm{Cz}$ (Figure 2 - top section). On the other hand, if the detection of alpha and beta rhythms over the sensorimotor cortex (those that reflect MI activity) is of interest, the typical recording sites are $\mathrm{C} 3, \mathrm{Cz}$ and $\mathrm{C} 4$, in conjunction with their neighboring sites (Figure 2 - bottom section).
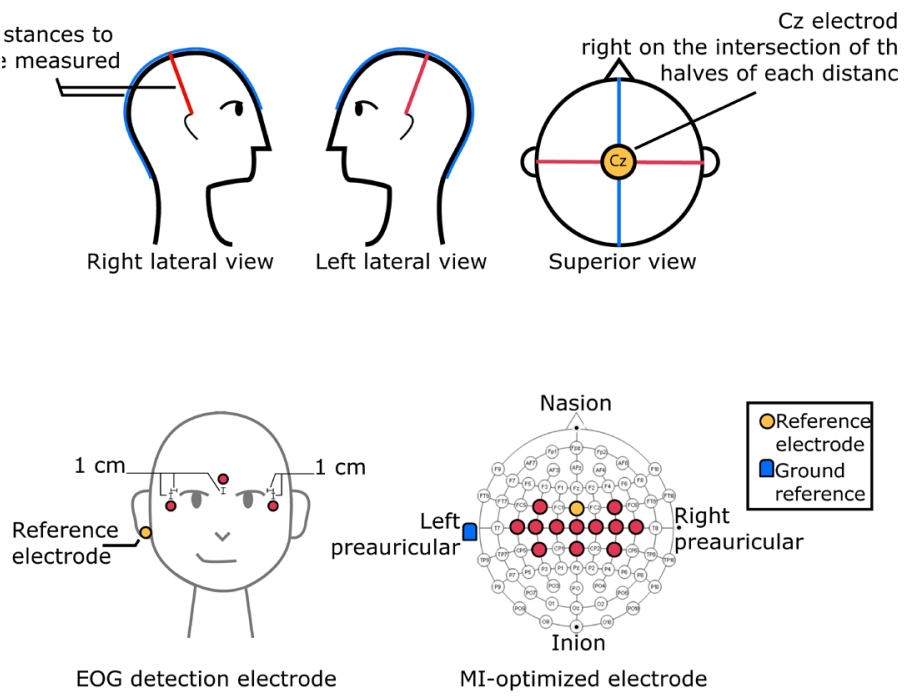

FIGURE 2. EEG and EOG electrode montage: procedure and location. EEG are mounted over the sensory-motor cortex to record $\mathrm{C} 3, \mathrm{Cz}$ and $\mathrm{C} 4$, along their neighboring recording sites to spatially filter by small Laplacian method.

\section{Removal of EOG artefacts}

Electrical activity proceeding from eye movements and blinking (i.e., electrooculography (EOG)) are one of the main interference sources during EEG recording. As a result, it is very important to adopt a method to minimize EOG effects. A practical and optimal method to detect and remove EOG artifacts in online mode was proposed in ${ }^{[35]}$. The EOG montage is based on three recording sites (Figure 2 - bottom section) and a three-minutes-long recording. Over the first two minutes, participants should be asked to follow with their sight an object covering as much sight space clockwise for the first minute, and counter-clockwise for the second one. Over the third minute, participants should be asked to blink continuously.

\section{Calibration and online communication}

As it is well-established, a minimum of 40 trials per control task in use is necessary to calibrate a MI based BCI. Once the system has been calibrated, brain-computer communication can be initiated. During BCI experiments, it is very important to keep in mind that volunteers need to take comfortable positions, to 
drink some water or eat some snack, to stand up and move around, or even to be on the phone from time to time. Relaxation periods are essential for any experimental protocol since human beings have limited mental resources ${ }^{[36]}$.

\section{User-Experience (UX)}

In addition to the individual characteristics of users, their perspective about the BCI system should be studied as well ${ }^{[37]}$. Precisely, the term "user-experience" was introduced to define a person perceptions and responses that result from the use of a system. According to human-centered design processes for interactive systems (ISO 9241-210), UX includes the emotions, beliefs, preferences, perceptions, physical and physiological responses, behaviors and accomplishments of the user before, during, and after the interaction with the system. UX evaluation might allow to redesign BCI prototypes in terms of user necessities and desires. An alternative can be the official UX questionnaire. The purpose of UX questionnaire is to evaluate aesthetic attractiveness, level of task understanding, predictability, levels of innovation, creativity and excitement. This source of information can be used to improve user-system interaction in BCIs. In ${ }^{[38]}$, it is suggested that the distribution of UX questionnaires in BCI studies has increased user acceptance, user enjoyment, and human-computer interaction. This evaluation could lead to increase the interaction quality between the user and the BCI system [39, 40].

\section{DISCUSSION}

A distinctive characteristic of BCI community during the system-part development has been the transparent and openness of the computational algorithms, what has led to the standardization of data processing pipelines. So far, a wide variety of open-source software has been released. The best known and extensively used packages include BCI2000 [41], BCILAB ${ }^{[42]}$, BioSig ${ }^{[43]}$, FieldTrip toolbox ${ }^{[44]}$, OpenViBE ${ }^{[45]}$, and EEGLAB ${ }^{[46]}$. In order to continue fostering the commu- nication practices ${ }^{[47]}$, it is proposed a step-by-step methodology towards the interactive prototyping of MI-based BCIs. According to Figure 1, the methodology involves 13 steps, which are encompassed under five stages: user-profile, psychological and neurophysiological assessment, brain-computer communication, and UX evaluation. See Figure 1.

Towards designing BCIs in terms of user-profiles, neurophysiological factors and UX, the first step that a $\mathrm{BCI}$ research team must take in order to create a new interaction paradigm relies exclusively on getting to know the user. This means to actually reach for the user by considering demographic factors, socioeconomic position, familiarity with technology products, occupation, devices and products which are constantly in contact with the user among many other factors that are often trivialized. User and his/her context represent a mine of information which can be used to take advantage of elements that could result familiar to them for designing a system which can merge easily with reality. The second step is to consider expectations from the user, which derive from the context. Questions such as (1) Has the user used a computer before? (2) Does the user perform long concentration tasks? If yes, (3) how does the user feel about that? (4) What are the concepts that come to the user mind when BCIs are mentioned to him? and (5) How much time does he/she think that he/she can maintain focus on repeating modulation tasks? must be answered before any line is drawn or any code is typed. The community must reject the idea of adapting the users to what is already there if a better performance wanted to be reached. The third and last step refers to the design and development the BCI system and an appropriate working environment. For that purpose, it must be considered that imaginary movements are predicted in line with motor repertoires built along lifetime, and sensory predictions made through context scanning (similar to actual movements). Therefore, a favorable and familiar system and working environment that 
provide at a first glance the sufficient sensory information about which imaginary movements are needed to interact with such environment must be created. Thereafter, it is necessary to identify the imaginary movements in line with the nature of the working environment so as to modify that environment as if imaginary movements were being actually executed. This achieves consistency between what is imagined and how that mental image is effectuated. Frequently, the set of imaginary movements that user performs to establish brain-machine communication is not strongly related to the control panel of the system. The consistency between imaginary movements and control mechanisms is referred to as transparent mapping. Finally, sensory feedback to obtain perceptual information about the environmental changes effected by the MI activity in use must be provided.

Prototyping interactive MI based BCIs in terms of user-profiles, psychological and neurophysiological factors, and UX can lead to the early involvement of end-users, and the appropriate contextualization of the final applicability of the system. At early stages of MI based BCI prototyping, this design strategy identifies and involves day-to-day activities, employment of existing technology, emotions, and expectations of end-users. Thereby, questions such as (1) What are the simplest and most routine tasks of users at the time of interacting with present-day technology? (2) How can those tasks be mapped into MI related control tasks? (3) Do users feel comfortable at using this kind of technological platforms? (4) What are the user expectations about the system performance? or (5) Would users make use of BCI based technology for their activities of daily living? could be answered. Based on that documentation, more feasible prototypes may result.

Interaction design of MI based BCIs challenges traditional paradigms since it attempts to restructure the system functionality by making users the central part of the system. This empathy-driven approach has shown to provide a better UX, which in turn diminishes frustration and increases involvement. Interaction design does not pretend to discard or demerit previous efforts, but to move towards natural and intuitive user-system interactions. This design technique may help users to imagine specific movements in specific contexts, facilitating the detection to brain patterns associated with the control tasks in use $[48,49,50,51,52]$. In the traditional paradigms, it is common practice to map arbitrarily imaginary movements of (1) mouth, (2) foot, (3) left hand, and (3) right hand to the control commands (1) move forward, (2) move backward, (3) turn left, and (4) turn right, regardless of the particular BCI application. Namely, what user thinks is not what system effectuates. This inconsistent control mapping causes confusion, and hinders the user-system adaptation, since not only MI skill acquisition is necessary, but also the correlation between mental rehearsal and control panel ${ }^{[53,54,55]}$. Interaction design allows to conceptualize and contextualize opinions, emotions, and expectations of end-users. The preliminary results presented in ${ }^{[50]}$ and ${ }^{[54]}$, along with the critiques provided ${ }^{[48]}$, are the theoretical framework of this proposal, and demonstrate the relevance of interaction design of MI based BCIs.

Regardless of the benefits of interaction design in MI based BCI prototype, there are some sources of weakness in the approach. Firstly, this practice is rarely implemented by the BCI community since it takes time and demands resources. Secondly, the appropriate implementation of interactive methodologies strongly determines the system performance. Thirdly, there is no a conventional methodology yet. Moreover, methodological standardization could be complicated since interaction design hinges on the application of the system. Interaction design is a heuristic approach since every person is different, and then, the prototype cannot be considered neither universally functional or accepted. 


\section{CONCLUSION}

The interest on MI based BCIs has been growing exponentially. Although the idea of direct brain-computer communication is very attractive stand alone, BCIs as a tool in Neurosciences to investigate sensorimotor transformations of the nervous system has magnified BCI research. Of particular interest is the neural mechanism behind the motor system, because movement is the only way human beings have for interacting with the world. When this system is malfunctioning, people eventually or suddenly lose their autonomy, what leads to overcome several socio-economical pitfalls. Only in Mexico, around 15.9 million people have some kind of limitation, either mental or physical. This means that $6 \%$ of the total population in the country have a poor quality of life. According to the National Institute of
Statistics and Geography (2014), mobility restrictions are the most recurrent disability and they are typically associated with aging process, traumatic injuries or congenital conditions. Unfortunately, MI based BCIs are still a laboratory prototype since not anyone at any time can control the system. The system functionality greatly depends on the modulation of EEG signals by means of MI related tasks. MI as control task in BCIs has been seen as a skill that must be acquired, but no user conditions nor controlled learning conditions have been taken into account. In this paper, it has been proposed a mixed methodology on the basis of (1) user-profiles, (2) psychological and neurophysiological factors associated with MI control tasks, and (3) UX so as to improve individual user performances, and increase the number of qualified users. 


\section{REFERENCES}

[1] C. S. Nam, A. Nijholt and F. Lotte, Brain-Computer Interfaces Handbook: Technological and Theoretical Advances, New York: CRC Press, 2018.

[2] C. Jeunet, B. N'Kaoua and F. Lotte, "Advances in user-training for mental-imagery-based BCI control: Psychological and cognitive factors and their neural correlates," Progress in Brain Research, vol. 228, no. 1, pp. 3-35, 2016.

[3] M. Ahn and S. C. Jun, "Performance variation in motor imagery brain-computer interface: A brief review," Journal of neuroscience methods, vol. 243, no. 1, pp. 103-110, 2015.

[4] S. Marchesotti, M. Bassolino, A. Serino, H. Bleuler and O. Blanke, "Quantifying the Role of Motor Imagery in Brain-Machine Interfaces," Scientific Reports, vol. 6, no. 1, p. 24076, 2016.

[5] M. Grosse-Wentrup and B. Schölkopf, "A review of performance variations in SMR-based Brain- Computer interfaces (BCIs)," BrainComputer Interface Research, vol. Springer Berlin Heidelberg, no. 1, pp. 39-51, 2013.

[6] J. Štastný, P. Sovka and M. Kostilek, "Overcoming Inter-Subject Variability In BCI Using EEG-Based Identification," Radioengineering, vol. 23, no. 1, 2014.

[7] Y. Rogers, et al., Interaction design: beyond human-computer interaction, 3rd ed., Italy: John Wiley \& Sons, 2011.

[8] E. G. Chrysikou, D. Casasanto and S. L. Thompson-Schill, "Motor experience influences object knowledge," Journal of Experimental Psychology: General, vol. 146, no. 3, p. 395, 2017.

[9] D. Benyon, Designing Interactive Systems: A comprehensive guide to HCI, UX and interaction design, 3rd ed., Edinburgh: Pearson, 2014.

[10] H. Gürkök, G. Hakvoort, M. Poel and A. Nijholt, "Meeting the Expectations from Brain-Computer Interfaces," Computers in Entertainment, vol. 15, no. 3, pp. 1-10, 2017.

[11] T. W. Picton, S. Bentin, P. Berg, E. Donchin, S. A. Hillyard, R. Johnson, G. A. Miller, W. Ritter, D. S. Ruchkin, M. D. Rugg and M. J. Taylor, "Guidelines for using human event-related potentials to study cognition: recording standards and publication criteria," Psychophysiology, vol. 37, no. 2, pp. 127-152, 2000.

[12] C. Jeunet, B. N'Kaoua and F. Lotte, Towards a cognitive model of mi-bci user training, Bordeaux: https://hal.archives-ouvertes.fr/hal01519476, 2017.

[13] L. M. Alonso-Valerdi and A. A. González-Garrido, "Cognitive and Computational Neuroscience - Principles, Algorithms and Applications," in Characterizing Motor System to Improve Training Protocols Used in Brain-Machine Interfaces Based on Motor Imagery, S. A. Hosseini, Ed., DOI: 10.5772/intechopen.68377, InTech Open, 2018, pp. 57-78

[1]4 W. Klimesch, "An algorithm for the EEG frequency architecture ofconsciousness and brain body coupling," Frontiers in Human Neuroscience, vol. 7, pp. 1-4, 2013.

[15] R. J. Gentili and C. Papaxanthis, "Laterality effects in motor learning by mental practice in right-handers," Neuroscience, vol. 297, no. 1, pp. 231-242, 2015.

[16] R. Oldfield, "The assessment and analysis of handedness: the Edinburgh inventory," Neuropsychologia, vol. 9, no. 1, pp. 97-113, 1971.
[17] E. M. Hammer, S. Halder, B. Blankertz, B. Sannelli, T. Dickhaus, S. Kleih, K. R. Müller and A. Kübler, "Psychological predictors of SMRBCI performance," Biological Psychology, vol. 89, no. 1, pp. 80-86, 2012.

[18] C. Jeunet and F. Lotte, "Why and How to Use Intelligent Tutoring Systems to Adapt MI-BCI Training to Each User," in 6th International BCI Meeting, Graz, 2016.

[19] A. Myrden and T. Chau, "Effects of user mental state on EEG-BCI performance," Frontiers in Human Neurosciences, vol. 9, p. 308, 2015.

[20] M. Gregg, C. Hall and A. Butler, "The MIQ-RS: a suitable option for examining movement imagery ability," Evidence-Based Complementary and Alternative Medicine, vol. 7, no. 2, pp. 249257, 2010.

[21] F. Malouin , C. L. Richards, P. L. Jackson, M. F. Lafleur, A. Durand and J. Doyon, "The Kinesthetic and Visual Imagery Questionnaire (KVIQ) for assessing motor imagery in persons with physical disabilities: a reliability and construct validity study," Journal of Neurologic Physical Therapy, vol. 31, no. 1, pp. 20-29, 2007.

[22] C. Papadelis, C. Kourtidou-Papadeli, P. Bamidis and M. Albani, "Effects of imagery training on cognitive performance and use of physiological measures as an assessment tool of mental effort," Brain and Cognition, vol. 64, no. 1, pp. 74-85, 2007.

[23] A. Vuckovic and B. A. Osuagwu, "Using a motor imagery questionnaire to estimate the performance of a brain-computer interface based on object oriented motor imagery," Clinical Neurophysiology, vol. 124, no. 1, pp. 1586-1595, 2013.

[24] B. Blankertz, C. Sannelli, S. Halder, A. Kubler, K. R. Muller, G. Curio and T. Dickhaus, "Neurophysiological predictor of SMR-based BCI performance," Neuroimage, vol. 51, no. 4, pp. 1303-1309, 2010.

[25] M. Grosse-Wentrup and B. Scholkopf, "High gamma-power predicts performance in sensorimotor-rhythm brain-computer interfaces," Journal of Neural Engineering, vol. 9, no. 046001, 2012.

[26] A. Guillot, C. Collet, V. A. Nguyen, F. Malouin, C. Richards and J. Doyon, "Functional neuroanatomical networks associated with expertise in motor imagery," Neuroimage, vol. 41, no. 4, pp. 14711483, 2008.

[27] D. Posthuma, M. C. Neale, D. I. Boomsma and E. J. C. de Geus, "Are smarter brains running faster? Heritability of alpha peak frequency, IQ, and their interrelation," Behavior Genetics, vol. 31, no. 6, pp. 567-79, 2001.

[28] S. Halder, D. Agorastos, R. Veit, M. Eva, S. Hammer, B. Lee, B. Varkuti, M. Bogdan, W. Rosenstiel, N. Birbaumer and A. Kübler, "Neural mechanisms of brain-computer interface control," Neuroimage, pp. 1779-1790, 2011.

[29] L. M. Alonso-Valerdi, "Python Executable Script for Estimating Two Effective Parameters to Individualize Brain-Computer Interfaces: Individual Alpha Frequency and Neurophysiological Predictor," Frontiers in Neuroinformatics, vol. doi: 10.3389/ fninf.2016.00022, pp. 10-22, 2016.

[30] T. K. Bera, "Noninvasive electromagnetic methods for brain monitoring: a technical review," Brain-Computer Interfaces, vol. Springer, no. Cham, pp. 51-95, 2015. 
[31] J. Dien, "Dien J. Issues in the application of the average reference: Review, critiques, and recommendations," Behavior Research Methods, Instruments, \& Computers, vol. 30, no. 1, pp. 34-43, 1998.

[32] F. F. Offner, "The EEG as potential mapping: the value of the average monopolar reference," Electroencephalography and clinical neurophysiology, vol. 2, no. 1, pp. 213-214, 1950.

[33] J. W. Osselton, "Acquisition of EEG data by bipolar unipolar and average reference methods: a theoretical comparison," Clinical Neurophysiology, vol. 19, no. 5, pp. 527-528, 1965.

[34] S. H. Choi, M. Lee, Y. Wang and B. Hong, "Estimation of optimal location of EEG reference electrode for motor imagery based BCI using fMRI," New York, 2006.

[35] A. Schlögl, C. Keinrath, D. Zimmermann, R. Scherer, R. Leeb and G. Pfurtscheller, "A fully automated correction method of EOG artifacts in EEG recordings," Clinical Neurophysiology, vol. 118, no. 1, pp. 98-104, 2007.

[36] D. A. Norman and D. G. Bobrow, "On data-limited and resourcelimited processes," Cognitive psychology, vol. 7, no. 1, pp. 44-64, 1975

[37] H. Gürkök, D. Plass-Oude Boss, B. L. Laar, F. Nijboer and A. Nijholt, "User experience evaluation in BCI: Filling the gap," International Journal of Bioelectromagnetism, vol. 13, no. 1, pp. 54-55, 2011.

[38] D. Plass-Oude Bos, H. Gürkök, B. Van de Laar, F. Nijboer and A. Nijholt, "User Experience Evaluation in BCI: Mind the Gap," International Journal of Bioelectromagnetism, vol. 13, no. 1, pp. 48-49, 2011.

[39] H. B. Santoso, M. Schrepp, R. Isal, A. Y. Utomo and B. Priyogi, "Measuring User Experience of the Student-Centered e-Learning Environment," Journal of Educators Online, vol. 13, no. 1, pp. 58-79, 2016.

[40] M. Schrepp, A. Hinderks and J. Thomaschewski, "Applying the User Experience Questionnaire (UEQ) in different evaluation scenarios," International Conference of Design, User Experience, and Usability, vol. Springer, no. Cham, pp. 383-392, 2014.

[41] J. Mellinger and G. Schalk, "BCI2000: A general-purpose software platform for BCI," in Toward Brain-Computer Interfacing, Cambridge, MIT Press, 2007, pp. 359-368.

[42] C. A. Kothe and S. Makeig, "BCILAB: a platform for brain-computer interface development," Journal of Neural Engineering, vol. 10, no. 5, p. 056014, 2013.

[43] A. Schlögl, C. Brunner, R. Scherer and A. Glatz, "BioSig: An opensource software library for BCI research," in Toward BrainComputer Interfacing, Cambridge, MIT Press, 2007, pp. 347-358.

[44] R. Oostenveld, P. Fries, E. Maris and J. M. Schoffelen, FieldTrip: open source software for advanced analysis of MEG, EEG, and invasive electrophysiological data, 1: Computational intelligence and neuroscience, 2011.
[45] Y. Renard, F. Lotte, G. Gibert, M. Congedo, E. Maby, V. Delannoy, O. Bertrand and A. Lécuyer, "OpenViBE: An open-source software platform to design, test, and use brain-computer interfaces in real and virtual environments," Presence: teleoperators and virtual environments, vol. 19, no. 1, pp. 35-53, 2010.

[46] A. Delorme and S. Makeig, "EEGLAB: an open source toolbox for analysis of single-trial EEG dynamics including independent component analysis," Journal of Neuroscience Methods, vol. 134, pp. 9-21, 2004.

[47] R. O. Gilmore, M. T. Diaz, B. A. Wyble and T. Yarkoni, Progress toward openness, transparency, and reproducibility in cognitive neuroscience, 1-14: Annals of the New York Academy of Sciences, 2017, pp. 1-14.

[48] R. Chavarriaga, M. Fried-Oken, S. Kleih and F. Lotte, "Heading for new shores! Overcoming pitfalls in BCI," Brain-Computer Interfaces, Taylor \& Francis, vol. IV, no. 1, pp. 60-73, 2016.

[49] A. Kübler, E. M. Holz, A. Riccio, C. Zickler, T. Kauffmann, S. C. Kleih, P. Staiger-Sälzer, L. Desideri, E.-J. Hoogerwerf and D. Mattia, "The User-Centered Design as Novel Perspective for Evaluating the Usability of BCI-Controlled Applications," Plos One, vol. 9, no. 12, 2014.

[50] Z. Qiu, B. Z. Allison, J. Jing, Y. Zhang, X. Wang, W. Li and A. Cichocki, "Optimized motor imagery paradigm based on imagining Chinese characters writing movement," IEEE Transactions on Neural Systems and Rehabilitation Engineering, vol. PP, no. 99, p. $1,2017$.

[51] F. Merienne, "Human factors consideration in the interaction process with virtual environment," International Journal on Interactive Design and Manufacturing, vol. 4, no. 2, pp. 83-86, 2010.

[52] J. M. D. Lynn, E. Armstrong and S. Martin, "User centred design and validation during the development of domestic brain computer interface applications for people with acquired brain injury and therapists: a multi-stakeholder approach," Journal of Assistive Technologies, vol. 10, no. 2, pp. 67-78, 2016.

[53] C. Jeunet, E. Jahanpour and F. Lotte, "Why standard braincomputer interface (BCI) training protocols should be changed: an experimental study," Journal of Neural Engineering, vol. 13, no. 3 , p. $036024,2016$.

[54] J. Schumacher, C. Jeunet and F. Lotte, "Towards Explanatory Feedback for User Training in Brain-Computer Interfaces," in Systems, Man, and Cybernetics (SMC), 2015 IEEE International Conference on, Kowloon, China, 2015.

[55] M. Schreuder, A. Riccio, M. Risetti, S. Dähne, A. Ramsay, J. Williamson, D. Mattia and M. Tangermann, "User-centered design in brain-computer interfaces-A case study," Artificial Intelligence in Medicine, vol. 2013, no. 59, pp. 71-80, 2013. 